Pacific Journal of Mathematics

CONVERGENT SOLUTIONS OF ORDINARY LINEAR 


\title{
CONVERGENT SOLUTIONS OF ORDINARY LINEAR HOMOGENEOUS DIFFERENCE EQUATIONS
}

\author{
W. J. A. Culmer and W. A. Harris, JR.
}

1. Introduction. This paper is concerned with the difference equation

$$
x(s+1)=A(s) x(s)
$$

where the 2 by 2 matrix $A(s)$ has a convergent series representation

$$
A(s)=s^{h} \sum_{k=0}^{\infty} A_{k} s^{-k}, \quad|s|>s_{0}
$$

and $A_{0} \neq 0$. The $A_{k}$ are constant matrices, the independent variable $s$ is complex, $h$ is a constant, and $x(s)$ is a column vector. We seek two independent vector solutions or a fundamental solution to the corresponding matrix equation

$$
X(s+1)=A(s) X(s) .
$$

The number of linearly independent solutions is not apparent since for both $A(s) \equiv 0$ and $A(s)=\left(\begin{array}{ll}0 & 0 \\ 1 & 0\end{array}\right)(1.2)$ has only $x(s) \equiv 0$ as a solution. We will show that there will be two linearly independent solutions unless the determinant of $A(s)$ vanishes identically, in which case there will either be one or none.

We begin, in $\S 2$, by reducing the matrix $A(s)$ to one of eight canonical forms which (after factoring out $s^{h^{\prime}}$ ) have convergent expansions in $s^{-1}$ or $s^{-1 / 2}$.

In $\S 3$ we construct formal solutions for these difference equations by substitution and direct comparison. The formal solutions will contain in general divergent power series, but it is expected that these formal solutions are asymptotic representations of true solutions in appropriate regions of the $s$-plane. Section 4 is devoted to estimates on the growth of the coefficients in these formal series.

In $\S 5$ we consider integral equations for vector functions $w(t)$ whose Laplace transforms are simply related to certain formal series occurring in our formal solutions. Using the estimates of $\S 4$ (in all but one case) we obtain true solutions of these integral equations.

These vector functions $w(t)$ are used in $\S \S 6$ and 7 to construct true solutions of the original difference equation. Theorems of Doetsch and Nörlund are utilized to prove that the formal solutions obtained

Received November 20, 1962. Supported in part by National Science Foundation Grant G-18918. 
earlier are asymptotic representations of these solutions. Moreover, these formal solutions may be summed and replaced by convergent factorial series in appropriate half-planes of the s-plane.

The relationship of this work to other results is discussed in $\S 8$.

This paper is based in part upon the Ph. D. thesis of W. J. A. Culmer under the direction of Professor H. L. Turrittin. Both authors are deeply indebted to their former teacher, Professor H. L. Turrittin, under whose guidance they first studied difference equations.

2. Canonical forms. If the elements of the matrix $P(s)$ are meromorphic in a neighborhood of $s=\infty$ and $\operatorname{det} P(s) \not \equiv 0$, the transformation

$$
x(s)=P(s) y(s)
$$

changes the difference equation (1.1) into

$$
y(s+1)=B(s) y(s)
$$

where

$$
B(s)=P^{-1}(s+1) A(s) P(s)=s^{h^{\prime}}\left(B_{0}+\frac{1}{s} B_{1}+\cdots\right), \quad|s|>s_{1} .
$$

Here $B(s)$ and $A(s)$ have qualitatively the same form. To facilitate the computation of formal solutions of the difference equation (1.1) we utilize transformations of the type (2.1) to achieve a more amenable form (2.2).

Our first goal is to diagonalize the leading term $B_{0}$ in the resulting expansion (2.3). This is not always possible with transformations $P(s)$ whose elements are meromorphic in some neighborhood of $s=\infty$. However, we give a necessary and sufficient condition for such a reduction. When it is not possible to achieve this diagonalization with a matrix $P(s)$ with meromorphic elements, we show that it is possible to diagonalize the leading term of $B(s)$ if we allow the elements of $P(s)$ to be meromorphic functions of $s^{1 / 2}$. In this case the elements of $s^{-h^{\prime}} B(s)$ will be meromorphic function of $s^{1 / 2} \cdot^{1}$ For these cases we are led to construct formal solutions containing formal power series in $s^{-1 / 2}$.

Let $J$ be a constant matrix such that $J^{-1} A_{0} J$ has Jordan canonical form

$$
J^{-1} A_{0} J=\left(\begin{array}{cc}
\rho_{1} & 0 \\
\beta & \rho_{2}
\end{array}\right)
$$

where $\beta=0$, if $\rho_{1} \neq \rho_{2}$. The transformation $B(s)=J^{-1} A(s) J$ is such that $B_{j}=J^{-1} A_{j} J$. Hence $B_{0}$ has diagonal form or the form

${ }^{1}$ It is one of these cases that we are unable to sum. 


$$
B_{0}=\left(\begin{array}{ll}
\rho & 0 \\
1 & \rho
\end{array}\right)
$$

For this case we have

Theorem 1. Let $B(s)$ and $C(s)$ be 2 by 2 matrices whose elements are analytic in some neighborhood of $s=\infty$,

$$
B(s)=\sum_{k=0}^{\infty} B_{k} s^{-k}, \quad C(s)=\sum_{k=0}^{\infty} C_{k} s^{-k},
$$

and let $B_{0}=\left(\begin{array}{ll}\rho & 0 \\ 1 & \rho\end{array}\right) . \quad A$ necessary and sufficient condition that there exist a matrix $P(s)$ with elements meromorphic in some neighborhood of $s=\infty$, det $P(s) \not \equiv 0, P^{-1}(s+1) B(s) P(s)=C(s)$, such that $C_{0}=\alpha I$ is that

$$
\left.\left\{\frac{1}{s} \operatorname{det}[\lambda I+s(B(s)-\rho I)]\right\}\right|_{s=\infty} \equiv 0
$$

Further, $\alpha=\rho$.

Proof. Let $B_{1}=\left(b_{i j}^{\prime}\right)$. A short calculation shows that

$$
\left.\left\{\frac{1}{s} \operatorname{det}[\lambda I+s(B(s)-\rho I)]\right\}\right|_{s=\infty}=b_{12}^{\prime} .
$$

Assume $b_{12}^{\prime}=0$. Choosing

$$
P(s)=\left(\begin{array}{cc}
s^{-1} & 0 \\
0 & 1
\end{array}\right)
$$

and writing $B(s)=\left(b_{i j}(s)\right)$, we have

$$
\begin{aligned}
C(s) & =P^{-1}(s+1) B(s) P(s)=\left(\begin{array}{cc}
\left(1+\frac{1}{s}\right) b_{11}(s) & (s+1) b_{12}(s) \\
\frac{1}{s} b_{21}(s) & b_{22}(s)
\end{array}\right) \\
& =\rho I+\frac{1}{s} C_{1}+\frac{1}{s^{2}} C_{2}+\cdots
\end{aligned}
$$

To prove the necessity we need a generalization of the fact that a function $f(s) \not \equiv 0$ meromorphic in some neighborhood of $s=\infty$ can be written in the form $f(s)=s^{\alpha} g(s)$ where $g(s)$ is analytic at $s=\infty$ and $g(\infty) \neq 0$.

Lemma 1. Let the elements of $P(s)$ be meromorphic in some neighborhood of $s=\infty, P(s)=\sum_{v=-k}^{\infty} P_{\nu} s^{-\nu}, \operatorname{det} P(s) \not \equiv 0, P_{-k} \neq 0$, and 
$k$ some finite integer. Then $P(s)$ can be represented in the form

$$
P(s)=U(s) s^{\alpha} V(s)
$$

where $V(s)$ is a polynomial in $s^{-1}$ with $\operatorname{det} V(s) \equiv 1, U(s)$ is a convergent power series in $s^{-1}$ with det $U(\infty) \neq 0$, and $s^{\alpha}=\left(\begin{array}{ll}s^{\alpha_{1}} & 0 \\ 0 & s^{\alpha_{2}}\end{array}\right)$, $\alpha_{1} \geqq \alpha_{2}$ integers.

Proof of Lemma 1. Consider $P(s)=\left(p_{1}(s), p_{2}(s)\right)$ where $p_{i}(s)$ are column vectors. Since $P_{-l} \neq 0$, choosing $V_{0}=I$ or $\left(\begin{array}{rr}0 & 1 \\ -1 & 0\end{array}\right)$ we may write

$$
P(s) V_{0}=\left(s^{-k} \hat{p}_{1}(s), s^{-k} \hat{p}_{2}(s)\right)
$$

where $\hat{p}_{i}(s)$ are power series in $s^{-1}$ and $\hat{p}_{1}(\infty) \neq 0$.

If $\hat{p}_{1}(\infty)$ and $\hat{p}_{2}(\infty)$ are linearly independent, choosing $U(s)=$ $\left(\hat{p}_{1}(s), \hat{p}_{2}(s)\right), \alpha_{1}=\alpha_{2}=-k$, and $V(s)=V_{0}^{-1}$ we have the representation (2.7). Using a sequence of matrices $V_{j}$,

$$
V_{j}=\left(\begin{array}{cc}
1 & c_{j} s^{-j+1} \\
0 & 1
\end{array}\right)
$$

we can write $P(s) V_{0} V_{1} \cdots V_{\beta}=\left(s^{-k} \hat{p}_{1}(s), s^{-k-\beta} \hat{p}_{2+\beta}(s)\right)$ choosing the first $\beta$ for which $\hat{p}_{1}(\infty)$ and $\hat{p}_{2+\beta}(\infty)$ are linearly independent. Indeed, since $\operatorname{det} P(s) \not \equiv 0, \quad \operatorname{det} P(s)=s^{-\gamma}\left(d_{0}+(1 / s) d_{1}+\cdots\right), \quad d_{0} \neq 0, \quad$ and $\operatorname{det} V_{j} \equiv 1, \beta=\gamma-2 k$.

Thus, choosing $U(s)=\left(\hat{p}_{1}(s) \hat{p}_{2+\beta}(s)\right), V(s)=\left(V_{0} V_{1} \cdots V_{\beta}\right)^{-1}, \alpha_{1}=$ $-k$ and $\alpha_{2}=-k-\beta$, we have $P(s)=U(s) s^{\alpha} V(s)$ and the lemma is proved.

Now assume that there exists a matrix $P(s)$ with elements meromorphic in some neighborhood of $s=\infty, \quad \operatorname{det} P(s) \not \equiv 0$ and $P^{-1}(s+1) B(s) P(s)=C(s)$ where $B(s)=\sum_{k=0}^{\infty} B_{k} s^{-k}, B_{0}=\left(\begin{array}{ll}\boldsymbol{\rho} & 0 \\ 1 & \rho\end{array}\right), C(s)=$ $\sum_{k=0}^{\infty} C_{k} s^{-k}, C_{0}=\alpha I$. By Lemma $1, P(s)$ has the representation (2.7) and defining

$$
\begin{aligned}
& \hat{C}(s)=\left(\frac{s+1}{s}\right)^{\alpha} V(s+1) C(s) V^{-1}(s)=V_{0} C_{0} V_{0}^{-1}+0\left(\frac{1}{s}\right) \\
& \hat{B}(s)=P(s) P^{-1}(s+1) B(s)=B_{0}+0\left(\frac{1}{s}\right)
\end{aligned}
$$

we have

$$
P^{-1}(s)[\hat{B}(s)-\rho I] P(s)=s^{-\alpha}[\hat{C}(s)-\rho I] s^{\alpha},
$$

and

$$
\operatorname{det}[\lambda I+\hat{B}(s)-\rho I]=\operatorname{det}[\lambda I+\hat{C}(s)-\rho I] .
$$


Thus, evaluating both sides of (2.8) for $s=\infty$ we have

$$
\lambda^{2}=(\lambda+\alpha-\rho)^{2}, \quad \text { or } \quad \alpha=\rho .
$$

Similarly,

$$
\frac{1}{s} \operatorname{det}[\lambda I+s(\hat{B}(s)-\rho I)]=\frac{1}{s} \operatorname{det}[\lambda I+s(\widehat{C}-\rho I)] .
$$

Since $C_{0}=\rho I, \operatorname{det}[\lambda I+s(\hat{C}(s)-\rho I)]=0(1)$, and

$$
\left.\left\{\frac{1}{s} \operatorname{det}[\lambda I+s(\hat{B}(s)-\rho I)]\right\}\right|_{s=\infty}=0 .
$$

This determinant depends only on the first two coefficients in the expansion of $\hat{B}(s)$. However, $P(s) P^{-1}(s+1)=I+0\left(s^{-2}\right)$, and hence

$$
\left.\left\{\frac{1}{s} \operatorname{det}[\lambda I+s(B(s)-\rho I)]\right\}\right|_{s=\infty} \equiv 0,
$$

and the theorem is proved.

REMARK. We have also proved that if the elements of $B(s)$ and $C(s)$ are analytic in some neighborhood of $s=\infty$ and are related by a matrix $P(s)$ with elements meromorphic in some neighborhood of $s=\infty, P^{-1}(s+1) B(s) P(s)=C(s), B_{0} \neq 0$,

$$
\operatorname{det}\left(\lambda I+B_{0}\right)=\operatorname{det}\left(\lambda I+C_{0}\right) .
$$

Returning now to our canonical form (2.5), we see that if $\rho \neq 0$, and $b_{12}^{\prime}=0$ we have diagonalized the leading coefficient. If $\rho=0$, and $b_{12}^{\prime}=0$, the problem is reduced to a similar problem with $h$ decreased by one. We may repeat this process until we achieve a problem for which the leading coefficient is a diagonal nonzero matrix or (1) the process does not terminate; or

(2) reach a stage for which Theorem 1 shows it is impossible to diagonalize the leading coefficient with meromorphic $P(s)$.

(1). If the process does not terminate, we have $C(s)=$ $P^{-1}(s+1) B(s) P(s)$ and in some neighborhood of $s=\infty, \operatorname{det} B(s)=$ $\operatorname{det} C(s) \operatorname{det} P(s+1)[\operatorname{det} P(s)]^{-1}=0\left(s^{h-k}\right)$ for arbitrarily large $k$. Hence $\operatorname{det} B(s) \equiv 0$ in some neighborhood of $s=\infty$.

Let $B(s)=s^{h}\left(b_{i j}(s)\right), b_{21}(\infty)=1$, $\operatorname{det} B(s) \equiv 0$. Then, the transformation matrix

$$
P(s)=\left(\begin{array}{cc}
1 & -\frac{b_{22}(s)}{b_{21}(s)} \\
0 & 1
\end{array}\right)
$$


is such that

$$
C(s)=P^{-1}(s+1) B(s) P(s)=\left(\begin{array}{ll}
c_{11}(s) & 0 \\
c_{21}(s) & 0
\end{array}\right), c_{21}(s)=b_{21}(s) .
$$

If $c_{11}(s) \not \equiv 0,{ }^{2}$ the transformation

$$
T(s)=\left(\begin{array}{cc}
1 & 0 \\
\frac{c_{21}(s)}{c_{11}(s-1)} & 1
\end{array}\right)
$$

is such that $\hat{C}=T^{-1}(s+1) C(s) T(s)=\left(\begin{array}{cc}c_{11}(s) & 0 \\ 0 & 0\end{array}\right)$ and the difference equation $y(s+1)=C(s) y(s)$ will have one nonzero solution.

If $c_{11}(s)=b_{11}(s) / b_{12}(s)+b_{22}(s+1) / b_{12}(s+1) \equiv 0$, the difference equation will have no nonzero solution.

(2). If we cannot diagonalize the leading coefficient with a meromorphic transformation $P(s)$ we arrive at a stage for which

$$
B(s)=P^{-1}(s+1) A(s) P(s)=s^{h^{\prime}}\left\{B_{0}+\frac{1}{s} B_{1}+\cdots\right\}
$$

where

$$
B_{0}=\left(\begin{array}{ll}
\rho & 0 \\
1 & \rho
\end{array}\right), B_{1}=\left(b_{i j}^{\prime}\right), b_{12}^{\prime} \neq 0 .
$$

The transformation

$$
T(s)=\left(\begin{array}{cc}
s^{-1 / 2} & 0 \\
0 & 1
\end{array}\right) J
$$

can be utilized to yield

$$
C(s)=T^{-1}(s+1) B(s) T(s)=s^{h^{\prime}}\left(C_{0}+\frac{1}{s^{12}} C_{1}+\frac{1}{s} C_{2}+\cdots\right)
$$

where $C_{0}=\rho I, C_{1}=J^{-1}\left(\begin{array}{cc}0 & b_{12}^{\prime} \\ 1 & 0\end{array}\right) J=\left(\begin{array}{cc}\sqrt{b_{12}^{\prime}} & 0 \\ 0 & -\sqrt{b_{12}^{\prime}}\end{array}\right)^{3}$.

Thus, we have achieved our first goal; there exists a transformation $P(s)$ with elements meromorphic functions of $s$ in a neighborhood of $s=\infty$ for which

$$
B(s)=P^{-1}(s+1) A(s) P(s)=s^{h^{\prime}}\left(B_{0}+\frac{1}{s} B_{1}+\cdots\right), B_{0}=\left(\begin{array}{cc}
\rho_{1} & 0 \\
0 & \rho_{2}
\end{array}\right) ;
$$

${ }^{2}$ If $c_{11}(s) \not \equiv 0$, the process will terminate and we will have essentially the infinitely repetitive process treated on page 11 . This can be seen directly also by a detailed analysis.

3 There are two cases, $\rho=0$ and $\rho \neq 0$. It is $\rho \neq 0$ which we are unable to sum. 
or no such transformation exists, in which case there exists a transformation $P(s)$ with elements meromorphic functions of $s^{1 / 2}$ in a neighborhood of $s=\infty$ for which

$$
\begin{aligned}
B(s) & =P^{-1}(s+1) A(s) P(s)=s^{h^{\prime}}\left(B_{0}+\frac{1}{s^{1 / 2}} B_{1}+\frac{1}{s} B_{2}+\cdots\right), \\
B_{0} & =\left(\begin{array}{cc}
\rho_{1} & 0 \\
0 & \rho_{2}
\end{array}\right), \text { and if } \rho_{1}=\rho_{2}, B_{1}=\left(\begin{array}{cc}
r_{1} & 0 \\
0 & r_{2}
\end{array}\right), r_{1} \neq r_{2} .
\end{aligned}
$$

We wish to refine the preliminary canonical forms (2.9) and (2.10). We note that the transformation

$$
T_{n \alpha}=I+\frac{Q_{n}}{(s-1)^{n / \alpha}} \quad(\alpha=1 \text { or } 2)
$$

is such that $T_{n a}^{-1}$ exists for $|s|$ large and

$$
T_{n \alpha}^{-1}(s+1) B(s) T_{n \alpha}(s)=s^{h^{\prime}}\left(C_{0}+\frac{1}{s^{1 / \alpha}} C_{1}+\frac{1}{s^{2 / \alpha}} C_{2}+\cdots\right)
$$

where $C_{j}=B_{j}$ for $j=0,1, \cdots, n-1$ and

$$
C_{n}=B_{n}+B_{0} Q_{n}-Q_{n} B_{0}
$$

and if $B_{0}=\rho I$, then $C_{j}=B_{j} j=0,1, \cdots, n$ and

$$
C_{n+1}=B_{n+1}+B_{1} Q_{n}-Q_{n} B_{1} .
$$

If the characteristic roots of $B_{0}$ or $B_{1}$ are distinct, $Q_{n}$ may be chosen so that $C_{n}$ or $C_{n+1}$ is a diagonal matrix with the same diagonal elements as $B_{n}$ or $B_{n+1}$ respectively.

Hence we are able to construct a transformation $T(s)$ with the same properties as $P(s)$ such that for (2.9) we have

$$
\begin{aligned}
C(s) & =T^{-1}(s+1) B(s) T(s)=s^{h^{\prime}}\left(C_{0}+\frac{1}{s} C_{1}+\cdots\right) . \\
C_{0} & =\left(\begin{array}{cc}
\rho_{1} & 0 \\
0 & \rho_{2}
\end{array}\right), \rho_{1} \neq \rho_{2}, C_{1}=\left(\begin{array}{cc}
r_{1} & 0 \\
0 & r_{2}
\end{array}\right),
\end{aligned}
$$

and for $(2.10)$

$$
\begin{gathered}
C(s)=T^{-1}(s+1) B(s) T(s)=s^{h^{\prime}}\left(C_{0}+\frac{1}{s^{1 / 2}} C_{1}+\frac{1}{s} C_{2}+\cdots\right) \\
C_{0}=\left(\begin{array}{cc}
\rho_{1} & 0 \\
0 & \rho_{2}
\end{array}\right), C_{1}=\left(\begin{array}{cc}
r_{1} & 0 \\
0 & r_{2}
\end{array}\right), C_{2}=\left(\begin{array}{cc}
p_{1} & 0 \\
0 & p_{2}
\end{array}\right), C_{3}=\left(\begin{array}{cc}
q_{1} & 0 \\
0 & q_{2}
\end{array}\right)
\end{gathered}
$$

and either $\rho_{1}=-\rho_{2} \neq 0$, or $\rho_{1}=\rho_{2} \neq 0$ and $r_{1}=-r_{2} \neq 0$. 
If for (2.10) $\rho_{1}=\rho_{2}=\rho \neq 0$, then we may assume $B_{1}=\left(\begin{array}{cc}r_{1} & 0 \\ \beta & r_{2}\end{array}\right)$, $\beta=0$ if $r_{1} \neq r_{2}$. If the characteristic roots $r_{1}, r_{2}$ differ by an integer, $\operatorname{Re}\left\{r_{1}\right\}>\operatorname{Re}\left\{r_{2}\right\}$, then the transformation

$$
T_{e}=\left(\begin{array}{cc}
1 & 0 \\
0 & s^{-1}
\end{array}\right)
$$

is such that $C(s)=T_{e}^{-1}(s+1) B(s) T_{e}(s)=s^{h^{\prime}}\left(C_{0}+(1 / s) C_{1}+\cdots\right)$ where $C_{0}=\rho I$ and $C_{1}$ has characteristic roots $r_{1}$ and $r_{2}+1$. Hence we may assume without loss of generality that in (2.10), if $\rho_{1}=\rho_{2}=\rho \neq 0$, $B_{1}=\left(\begin{array}{cc}r_{1} & 0 \\ \beta & r_{2}\end{array}\right)$ and $r_{1}-r_{2} \neq \pm 1, \pm 2, \cdots, \beta=0$ if $r_{1} \neq r_{2}$.

In every case except $\rho_{1}=0, \rho_{2} \neq 0$ we have achieved the desired canonical form. We may utilize transformations of the form $T_{n 1}$ as given by (2.11) so that either

$$
C(s)=T^{-1}(s+1) B(s) T(s)=s^{h^{\prime}}\left(C_{0}+\frac{1}{s} C_{1}+\cdots\right)
$$

where

$$
C_{j}=\left(\begin{array}{cc}
0 & 0 \\
0 & c_{22}^{j}
\end{array}\right)(j=0,1,2, \cdots, m-1) \quad c_{22}^{0} \neq 0
$$

and

$$
C_{m}=\left(\begin{array}{cr}
c_{11}^{m} & 0 \\
0 & c_{22}^{m}
\end{array}\right), c_{11}^{m} \neq 0 ; \quad \text { or }
$$

(2) the process does not terminate.

If the process does not terminate, it is clear that $\operatorname{det} B(s)=$ $0\left(s^{h^{\prime}-k}\right)$ for arbitrarily large $k$ in a neighborhood of $s=\infty$ and hence $\operatorname{det} B(s) \equiv 0$.

Writing $B(s)=s^{h}\left(b_{i j}(s)\right), b_{22}(\infty)=1$, we see that the transformation

$$
P(s)=\left(\begin{array}{cc}
1 & \frac{b_{12}(s-1)}{c_{22}(s-1)} \\
\frac{-b_{21}(s)}{b_{22}(s)} & 1
\end{array}\right)
$$

is such that $P^{-1}(s+1) B(s) P(s)=\left(\begin{array}{cc}0 & 0 \\ 0 & c_{22}(s)\end{array}\right), c_{22}(s)=s^{h^{\prime}}\left(1+(1 / s) c_{1}^{22}+\cdots\right)$ for $|s|$ large. This problem reduces to the two scalar equations $w_{1}(s+1)=0, w_{2}(s+1)=c_{22}(s) w_{2}(s)$.

We summarize our results in 
THEOREM 2. Let the elements of the square matrix of order two $A(s)$ have convergent representations in a neighborhood of $s=\infty$,

$$
A(s)=s^{h}\left(A_{0}+\frac{1}{s} A_{1}+\cdots\right)|s|>s_{0},
$$

$A_{0}=0, h$ a constant. There exists a matrix $P(s)$, $\operatorname{det} P(s) \not \equiv 0$, such that either

(1) the elements of $P(s)$ are meromorphic functions of $s$ in a neighborhood of $s=\infty$ and

$$
B(s)=P(s+1)^{-1} A(s) P(s)=s^{h^{\prime}}\left(B_{0}+\frac{1}{s} B_{1}+\cdots\right),
$$

where

$$
B_{0}=\left(\begin{array}{cc}
\rho_{1} & 0 \\
0 & \rho_{2}
\end{array}\right), B_{1}=\left(\begin{array}{cc}
r_{1} & 0 \\
0 & r_{2}
\end{array}\right), 0 \neq \rho_{1} \neq \rho_{2} \neq 0,
$$

$$
B_{0}=\rho_{1} I, B_{1}=\left(\begin{array}{cc}
r_{1} & 0 \\
0 & r_{2}
\end{array}\right), \rho_{1} \neq 0, r_{1}-r_{2} \neq \pm 1, \pm 2, \cdots
$$

$$
B_{0}=\rho_{1} I, B_{1}=\left(\begin{array}{cc}
r_{1} & 0 \\
\delta & r_{1}
\end{array}\right), \rho_{1} \neq 0, \delta \neq 0 \text {, }
$$

$$
\begin{aligned}
B_{j} & =\left(\begin{array}{cc}
0 & 0 \\
0 & b_{22}^{j}
\end{array}\right), j=0,1, \cdots, m-1, b_{22}^{0}=\rho_{2} \neq 0 \\
B_{m} & =\left(\begin{array}{cc}
b_{11}^{m} & 0 \\
0 & b_{22}^{m}
\end{array}\right), b_{11}^{m}=\rho_{1} \neq 0,
\end{aligned}
$$

(v) $B(s)=\left(\begin{array}{cc}p(s) & 0 \\ 0 & 0\end{array}\right), p(s)=s^{h^{\prime}}\left(p_{0}+\frac{1}{s} p_{1}+\cdots\right), p_{0} \neq 0$ ，

(vi) $B(s)=\left(\begin{array}{cc}0 & 0 \\ q(s) & 0\end{array}\right), q(s)=s^{h^{\prime}}\left(q_{0}+\frac{1}{s} q_{1}+\cdots\right), q_{0} \neq 0$;

or

(2) the elements of $P(s)$ are meromorphic functions of $s^{1 / 2}$ in a neighborhood of $s=\infty$ and

$$
B(s)=P^{-1}(s+1) A(s) P(s)=s^{h^{\prime}}\left(B_{0}+\frac{1}{s^{1 / 2}} B_{1}+\frac{1}{s} B_{2}+\cdots\right),
$$

where

(vii) $\quad B_{0}=\left(\begin{array}{cc}-\rho_{1} & 0 \\ 0 & \rho_{1}\end{array}\right), \rho_{1} \neq 0, B_{1}, B_{2}$ and $B_{3}$ diagonal matrices,

(viii) $\quad B_{0}=\rho_{1} I, B_{1}=\left(\begin{array}{cc}r_{1} & 0 \\ 0 & -r_{1}\end{array}\right), \rho_{1} \neq 0, r_{1} \neq 0, B_{2}$ and $B_{3}$ diagonal matrices. 
REMARK. The only solution of the difference equation $y(s+1)=$ $B(s) y(s)$ with canonical form (vi) is $y(s) \equiv 0$. For canonical form (v) the solution of $y(s+1)=B(s) y(s)$ has only a nonzero first component which is the same as both components of the equation $y(s+1)=$ $\left(\begin{array}{cc}p(s) & 0 \\ 0 & p(s)\end{array}\right) y(s)$ which is a subcase of canonical form (ii). Hence we omit canonical forms (v) and (vi) in the remainder of this paper.

3. Formal solutions. We now determine formal solutions for the difference equation

$$
y(s+1)=B(s) y(s)
$$

where $B(s)$ has one of the canonical forms given in Theorem 2 .

Let $B(s)$ have canonical form (i). We seek a solution of (3.1) in the form

$$
y(s)=s^{h^{\prime} s} \rho^{s} s^{r} v(s)
$$

where $\rho$ and $r$ are constants and $v(s)$ is a formal power series in $s^{-1}$. Substituting (3.2) into (3.1), $v(s)$ must satisfy the difference equation

$$
v(s+1)=C(s) v(s),
$$

where

$$
C(s)=\left(1+\frac{1}{s}\right)^{-h^{\prime}(s+1)} \frac{1}{\rho}\left(1+\frac{1}{s}\right)^{-r} s^{-h^{\prime}} B(s) .
$$

Using the relationships

$$
\begin{aligned}
\left(1+\frac{1}{s}\right)^{-h^{\prime}(s+1)} & =e^{-h^{\prime}(s+1) l n(1+(1 / s))}=e^{-h^{\prime}}\left(1-\frac{h^{\prime}}{2 s}+\cdots\right), \\
\left(1+\frac{1}{s}\right)^{-r} & =\left(1-\frac{r}{s}+\frac{r(r+1)}{s^{2}}+\cdots\right),
\end{aligned}
$$

we have $C(s)=C_{0}+(1 / s) C_{1}+\cdots$, where

$$
C_{0}=\frac{e^{-h^{\prime}}}{\rho} B_{0}, C_{1}=\frac{e^{-h^{\prime}}}{\rho}\left[B_{1}-\left(\frac{h^{\prime}}{2}+r\right) B_{0}\right] .
$$

Choosing $\rho=e^{-h^{\prime}} \rho_{1}, \rho_{1} \neq 0$ and $r=\left(r_{1} / \rho_{1}\right)-\left(h^{\prime} / 2\right)$, we may write $C_{0}=$ $\left(\begin{array}{ll}1 & 0 \\ 0 & \bar{\rho}\end{array}\right), C_{1}=\left(\begin{array}{ll}0 & 0 \\ 0 & \bar{r}\end{array}\right), \bar{\rho} \neq 1$.

Substituting a power series in $s^{-1}$ for $v(s)$ into (3.3) and using

$$
v(s)=\sum_{k=0}^{\infty} v_{k} s^{-k}, v(s+1)=v_{0}+\sum_{k=1}^{\infty} \sum_{j=1}^{k}(-1)^{k-j}\left(\begin{array}{c}
k-1 \\
j-1
\end{array}\right) v_{j},^{4}
$$

$4\left(\begin{array}{c}p \\ q\end{array}\right)$ is the binomial coefficient $\left(\begin{array}{c}p \\ q\end{array}\right)=\frac{\Gamma(p+1)}{\Gamma(q+1) \Gamma(p+q+1)}, p \geqq q \geqq-1$. 
we have

$$
\begin{gathered}
\left(I-C_{0}\right) v_{0}=0,\left(I-C_{0}\right) v_{1}=C_{1} v_{0} \\
\left(I-C_{0}\right) v_{n}-\left((n-1) I-C_{1}\right) v_{n-1} \\
=\sum_{m=1}^{n-2}(-1)^{n-m}\left(\begin{array}{c}
n-1 \\
m-1
\end{array}\right) v_{m}+\sum_{m=2}^{n} C_{m} v_{n-m}
\end{gathered}
$$

The choice made for $\rho$ and $r$ allow us to solve the first equation (3.4) by any vectors of the form $v_{0}=\left(\begin{array}{c}v_{1}^{0} \\ 0\end{array}\right), v_{1}=\left(\begin{array}{c}v_{1}^{1} \\ 0\end{array}\right) . \quad v_{1}^{0}$ is arbitrary, say $v_{1}^{0}=1$ and $v_{1}^{1}$ and $v_{2}^{2}$ will be determined from (3.5) with $n=2$. In general $v_{1}^{k-1}$ and $v_{2}^{k}$ will be determined from (3.5) with $k=n$.

If $\rho_{2} \neq 0$, then choosing $\rho=e^{-h^{\prime}} \rho_{2}$ and $r=\left(r_{2} / \rho_{2}\right)-\left(h^{\prime} / 2\right)$ will give rise to a second solution. In this case $v_{0}=\left(\begin{array}{l}0 \\ 1\end{array}\right)$ and hence it is clear that the two formal solutions so determined will be (formally) linearly independent.

For the canonical form (ii), $B_{0}=\rho_{1} I, B_{1}=\left(\begin{array}{cc}r_{1} & 0 \\ 0 & r_{2}\end{array}\right), \rho_{1} \neq 0, r_{1}-r_{2} \neq$ $\pm 1, \pm 2, \cdots$ we choose $\rho=\rho_{1}$ and $r=\left(r_{1} / \rho_{1}\right)-\left(h^{\prime} / 2\right)$ to obtain $C_{0}=I$, $C_{1}=\left(\begin{array}{ll}0 & 0 \\ 0 & \bar{r}\end{array}\right), \bar{r}=\left(r_{2}-r_{1}\right) / \rho_{1}$. The equations for determining $v(s)$ are as before (3.4) and (3.5). If $\bar{r} \neq 0 \quad v_{0}=\left(\begin{array}{l}1 \\ 0\end{array}\right)$ will satisfy (3.4) and the $v_{n}, n \geqq 1$ are determined by (3.5) since $\left(I-C_{0}\right)=0$ and $(n-1) I-C_{1}$ is nonsingular for $n=2,3, \cdots$. When $\bar{r} \neq 0$, a second solution is obtained by choosing $r=\left(r_{2} / \rho_{1}\right)-\left(h^{\prime} / 2\right)$ and $v_{0}=\left(\begin{array}{l}0 \\ 1\end{array}\right)$. If $\bar{r}=0$, i.e. $r_{1}=r_{2}, v_{0}$ is arbitrary and choosing $v_{0}=\left(\begin{array}{l}1 \\ 0\end{array}\right)$ and $\left(\begin{array}{l}0 \\ 1\end{array}\right)$ will give two linearly independent formal solutions.

If we have canonical form (iii), then choosing $\rho=e^{-h^{\prime}} \rho_{1}, r=$ $\left(r / \rho_{1}\right)-\left(h^{\prime} / 2\right)$, we have $C_{0}=I, C_{1}=\left(\begin{array}{ll}0 & 0 \\ 1 & 0\end{array}\right)=E .{ }^{5}$ We note that $E$ is a nilpotent and $E^{2}=0$.

A simple calculation shows that if we can find two linearly independent vectors $v$ and $u$ such that

$$
\begin{aligned}
& v(s+1)=C(s) v(s) \\
& u(s+1)=C(s) u(s)-v(s+1) \ln \left(1+\frac{1}{s}\right),
\end{aligned}
$$

then $v(s)$ and $u(s)+v(s) l n s$ will be two linearly independent solutions for $v(s+1)=C(s) v(s)$ when $B(s)$ has canonical form (iii).

${ }^{5}$ Since $\delta$ was any nonzero constant, we assume $\delta=\frac{e^{-h^{\prime}}}{\rho_{1}}$ so that $C_{1}$ has the form shown. 
Substitution of a power series for $v(s)$ and an identification of terms yields

$$
\begin{aligned}
E v_{0} & =0,(I+E) v_{1}=-C_{2} v_{0} \\
(n I+E) v_{n} & =\sum_{j=2}^{n}\left[(-1)^{j}\left(\begin{array}{c}
n \\
j
\end{array}\right) I-C_{j}\right] v_{n-j+1}-C_{n+1} v_{0} .
\end{aligned}
$$

Hence choosing $v_{0}=\left(\begin{array}{l}0 \\ 1\end{array}\right), v_{1}=(E-I) C_{2} v_{0}$, and $v_{n}$ determined by (3.7) for $n=2,3, \cdots,\left((n I+E)^{-1}=(1 / n) I-\left(1 / n^{2}\right) E\right), v(s)$ will be determined.

Substituting a power series for $u(s)$ into the corresponding difference equation and expanding $\ln (1+(1 / s))$ as a power series in $s^{-1}$ we have

$$
\begin{gathered}
E u_{0}=v_{0}, \quad \text { or } \quad u_{0}=\left(\begin{array}{c}
1 \\
u_{2}^{0}
\end{array}\right) \\
u_{n}=\frac{1}{n}\left(\frac{E}{n}-I\right)\left\{\frac{(-1)^{n+1}}{n+1} v_{0}+C_{n+1} u_{0}+\sum_{j=2}^{n}\left[C_{j}-(-1)^{j}\left(\begin{array}{c}
n \\
j
\end{array}\right) I\right] u_{n-j+1}\right. \\
\left.+\sum_{k=1}^{n} \sum_{j=0}^{n-k} \frac{(-1)^{k+j}}{k}\left(\begin{array}{c}
n-k \\
j
\end{array}\right) v_{n+1-k-j}\right\} .
\end{gathered}
$$

Clearly these two vectors $v(s)$ and $u(s)+v(s) l n s$ are (formally) linearly independent since

$$
\operatorname{det}(v, u+v \operatorname{lns})=\operatorname{det}(v, u)=1+0\left(\frac{1}{s}\right) .
$$

If $B(s)$ has canonical form (iv), the technique used for canonical form (i) will yield one formal solution in the form

$$
y(s)=s^{h / s} \rho^{s} s^{r} v(s) .
$$

We seek a second solution in the form

$$
y(s)=s^{\left(h^{\prime}-m\right) s} \rho^{s} s^{r} v(s) .
$$

Hence corresponding to (3.3), $v(s)$ must satisfy

$$
v(s+1)=C(s) v(s),
$$

where

$$
\begin{aligned}
C(s) & =\left(1+\frac{1}{s}\right)^{-\left(h^{\prime}-m(s+1)\right)} \frac{1}{\rho}\left(1+\frac{1}{s}\right)^{-r} s^{m-h^{\prime}} B(s) \\
& =s^{m}\left(C_{0}+\frac{1}{s} C_{1}+\cdots\right) . \\
B_{j} & =\left(\begin{array}{cc}
0 & 0 \\
0 & b_{22}^{j}
\end{array}\right), j=0,1, \cdots, m-1, b_{22}^{0} \neq 0,
\end{aligned}
$$




$$
B_{m}=\left(\begin{array}{cc}
b_{11}^{m} & 0 \\
0 & b_{22}^{m}
\end{array}\right), b_{11}^{m} \neq 0, B_{m+1}=\left(\begin{array}{cc}
b_{11}^{m+1} & 0 \\
0 & b_{22}^{m+1}
\end{array}\right)
$$

imply

$$
\begin{aligned}
C_{j} & =\left(\begin{array}{cc}
0 & 0 \\
0 & c_{22}^{\jmath}
\end{array}\right), j=0,1, \cdots, m-1, c_{22}^{0}=\frac{e^{-\left(h^{\prime}-m\right)}}{\rho} b_{22}^{0} \\
C_{m} & =\left(\begin{array}{cc}
c_{11}^{m} & 0 \\
0 & c_{22}^{m}
\end{array}\right), c_{11}^{m}=\frac{1}{\rho} e^{-\left(h^{\prime}-m\right)} b_{11}^{m}, \\
C_{m \vdash 1} & =\left(\begin{array}{cc}
c_{1}^{m}+1 & 0 \\
0 & c_{22}^{m+1}
\end{array}\right), c_{11}^{m+1}=\frac{e^{-\left(h^{\prime}-m\right)}}{\rho}\left[b_{11}^{m+1}-\left(\frac{h^{\prime}-m}{2}+r\right) b_{11}^{m}\right] .
\end{aligned}
$$

Hence choosing $\rho=e^{-\left(h^{\prime}-m\right)} b_{11}^{m}$ and $r=\left[b_{11}^{m+1}-\left(\left(h^{\prime}-m\right) / 2\right) b_{11}^{m}\right] / b_{11}^{m}$, we have

$$
C_{m}=\left(\begin{array}{cc}
1 & 0 \\
0 & c_{22}^{m}
\end{array}\right), C_{m+1}=\left(\begin{array}{cc}
0 & 0 \\
0 & c_{22}^{m+1}
\end{array}\right), C_{0}=\left(\begin{array}{cc}
0 & 0 \\
0 & c_{22}^{0}
\end{array}\right), c_{22}^{0} \neq 0 .
$$

Substituting a power series for $v(s)$ into (3.11) yields

$$
\begin{aligned}
& \sum_{j=0}^{\nu} C_{\nu-j} v_{j}=0, \nu=0,1, \cdots, m-1, \sum_{j=0}^{m} C_{m-j} v_{j}=v_{0} \\
& \sum_{j=0}^{m+n} C_{m+n-j} v_{j}=\sum_{k=1}^{n}(-1)^{n+k}\left(\begin{array}{l}
n-1 \\
k-1
\end{array}\right) v_{k}, n=1,2, \cdots,
\end{aligned}
$$

Choosing $v_{j}=\left(\begin{array}{c}v_{1}^{j} \\ 0\end{array}\right)$ for $j=0, \cdots, m,(3.12)$ is satisfied and (3.13) can be written

$$
\begin{aligned}
C_{0} v_{n+m}+(n-1) v_{n-1}= & \left(I-C_{m}\right) v_{n}+\sum_{k=1}^{n-2}(-1)^{n-k}\left(\begin{array}{l}
n-1 \\
k-1
\end{array}\right) v_{k} \\
& -\sum_{j=0}^{n-1} C_{m+n-j} v_{j}-\sum_{j=1}^{m-1} C_{m-j} v_{n+j} .
\end{aligned}
$$

For canonical forms (vii) and (viii) we seek a solution of the form

$$
y(s)=s^{h^{\prime} s} \rho^{s} e^{\mu s^{1 / 2}} s^{r} e^{\nu s-1 / 2} v(s) .
$$

Thus $v(s)$ must satisfy $v(s+1)=C(s) v(s)$ where

$$
C(s)=\left(1+\frac{1}{s}\right)^{-h^{\prime}(s+1)} \frac{1}{\rho} e^{-\mu\left[(s+1)^{1 / 2}-s^{1 / 2}\right]}\left(1+\frac{1}{s}\right)^{-r} e^{-\nu\left[(s+1)^{-1 / 2}-s^{-1 / 2}\right]} s^{-h^{\prime}} B(s) .
$$

As before $C(s)$ is a power series in $s^{-1 / 2} ; \rho$ is chosen so that $C_{0}$ will have the form

$$
C_{0}=\left(\begin{array}{cc}
-1 & 0 \\
0 & 1
\end{array}\right)\left(\text { case }(\text { vii) }) \text { or } C_{0}=I(\text { case }(\text { viii }))\right.
$$


The factor $e^{-\mu\left[(s+1)^{1 / 2}-s^{1 / 2}\right]}=1-(\mu / 2) s^{-1 / 2}+\cdots$ allows us to choose one of the diagonal elements of $C_{1}$ to be zero. As before $(1+(1 / s))^{-r}$ allows us to choose one of the diagonal elements of $C_{2}$ to be zero and $e^{-\nu\left[(s+1)^{-1 / 2}-s^{-1 / 2}\right]}$ allows us to choose one of the diagonal elements of $C_{3}$ to be zero. Thus choosing $\rho, \mu, r$, and $\nu$ in succession we may write for canonical form (vii)

$$
\begin{aligned}
C(s)=\left(\begin{array}{cc}
-1 & 0 \\
0 & 1
\end{array}\right) & +\frac{1}{s^{1 / 2}}\left(\begin{array}{ll}
\bar{r} & 0 \\
0 & 0
\end{array}\right)+\frac{1}{s}\left(\begin{array}{ll}
p & 0 \\
0 & 0
\end{array}\right) \\
& +\frac{1}{s^{3 / 2}}\left(\begin{array}{ll}
q & 0 \\
0 & 0
\end{array}\right)+\frac{C_{4}}{s^{2}}+\cdots
\end{aligned}
$$

and for canonical form (viii)

$$
C(s)=I+\frac{1}{s^{1 / 2}}\left(\begin{array}{ll}
\bar{r} & 0 \\
0 & 0
\end{array}\right)+\frac{1}{s}\left(\begin{array}{ll}
p & 0 \\
0 & 0
\end{array}\right)+\frac{1}{s^{3 / 2}}\left(\begin{array}{ll}
q & 0 \\
0 & 0
\end{array}\right)+\frac{C_{4}}{s^{3 / 2}}+\cdots,
$$

$\bar{r} \neq 0$ in (3.18).

For canonical form (vii) we get two solutions: one by choosing $\rho=\rho_{1}$ and choosing the second diagonal element to be zero in $C_{1}, C_{2}$, and $C_{3}$ as shown in (3.17); and another by choosing $\rho=-\rho_{1}$ and choosing the first diagonal element in $C_{1}, C_{2}$, and $C_{3}$ to be zero.

For canonical form (viii) we get two solutions corresponding to the two choices for $\mu$ in (3.15), namely to make either the first or second diagonal element of $C_{1}$ equal to zero.

Substituting power series for $v(s)$ in the difference equation $v(s+1)=C(s) v(s)$ where $C(s)$ has the form (3.17) or (3.18) yields: for canonical form (vii), (3.17)

$$
\begin{gathered}
\left(C_{0}-I\right) v_{0}=0,\left(C_{0}-I\right) v_{1}+C_{1} v_{0}=0 \\
\left(C_{0}-I\right) v_{2 n}+C_{1} v_{2 n-1}+\left[C_{2}+(n-1) I\right] v_{2 n-2} \\
=\sum_{j=1}^{n-2}(-1)^{n-j}\left(\begin{array}{l}
n-1 \\
n-j
\end{array}\right) v_{2 j}-\sum_{j=0}^{2 n-3} C_{2 n-j} v_{j} \\
\left(C_{0}-I\right) v_{2 n+1}+C_{1} v_{2 n}+\left[C_{2}+\left(n-\frac{1}{2}\right) I\right] v_{2 n-1} \\
=\sum_{j=1}^{n-1}(-1)^{n-j}\left(\begin{array}{c}
n-1 / 2 \\
n-j+1
\end{array}\right) v_{2 j-1}-\sum_{j=0}^{2 n-2} C_{2 n-j+1} v_{j} ;
\end{gathered}
$$

and the canonical form (viii), (3.18)

$$
\begin{gathered}
C_{1} v_{0}=0 \\
C_{1} v_{2 n-1}+\left[C_{2}+(n-1) I\right] v_{2 n-2} \\
=-\sum_{j=0}^{2 n-3} C_{2 n-j} v_{j}+\sum_{j=1}^{n-2}(-1)^{n-j}\left(\begin{array}{c}
n-1 \\
j-1
\end{array}\right) v_{2 j}
\end{gathered}
$$




$$
\begin{aligned}
C_{1} v_{2 n} & +\left[C_{2}+\left(n-\frac{1}{2}\right) I\right] v_{2 n-1} \\
& =-\sum_{j=0}^{2 n-2} C_{2 n-j+1} v_{j}+\sum_{j=0}^{n-2}(-1)^{n-j}\left(\begin{array}{l}
n-\frac{1}{2} \\
n-j
\end{array}\right) v_{2 j+1} .
\end{aligned}
$$

In each case the coefficients $v_{0}, v_{1}, v_{2}$ may be determined in succession to give two linearly independent formal solutions.

We have thus found the appropriate number of linearly independent formal solutions for all canonical forms given in Theorem 2 .

4. Growth estimates. We seek now to estimate the rate of growth of the coefficients of $v(s)$ in order to discuss the asymptotic nature of these solutions and the possibility of summing $v(s)$ and replacing the formal power series with a convergent factorial series.

We note that $v(s)$ is a formal solution of an equation of the form $v(s+1)=C(s) v(s)$ for which $C(s)$ has a convergent power series representation in $s^{-1}$ or $s^{-1 / 2}$ for $|s|>a$. Hence we have the estimate

$$
\left|c_{i j}^{n}\right| \leqq M a^{n} \quad M>0 ; \quad a>0 .
$$

Consider the canonical form (i). Here the coefficients $v_{n}$ are determined by (3.4), (3.5) which can be written in component form, $v_{n}=\left(\begin{array}{l}v_{1}^{n} \\ v_{2}^{n}\end{array}\right)$,

$$
\begin{aligned}
(n-1) v_{1}^{n-1} & =\sum_{j=1}^{n-2}(-1)^{n-j}\left(\begin{array}{c}
n-1 \\
j-1
\end{array}\right) v_{1}^{j}-\sum_{j=2}^{n} \sum_{i=1}^{2} c_{1 i}^{j} v_{i}^{n-j} \\
(1-\bar{\rho}) v_{2}^{n} & =(n-1+\bar{r}) v_{2}^{n-1}-\sum_{j=1}^{n-2}(-1)^{n-j}\left(\begin{array}{c}
n-1 \\
j-1
\end{array}\right) v_{2}^{j}+\sum_{j=2}^{n} \sum_{i=1}^{2} c_{21}^{j} v_{2}^{n-j}
\end{aligned}
$$

$n \geqq 3$

with $v_{0}=\left(\begin{array}{l}1 \\ 0\end{array}\right), v_{2}^{1}=0, v_{1}^{1}=-c_{11}^{2}, v_{2}^{2}=c_{21} /(1-\bar{\rho})$ as initial conditions. Let $\lambda=|1-\bar{\rho}| \neq 0$ and $r=|\bar{r}|$.

The coefficients $w_{n}$ determined by

$$
\begin{aligned}
& (n-1) w_{1}^{n}=\sum_{j=1}^{n-2}\left(\begin{array}{c}
n-1 \\
j-1
\end{array}\right) w_{1}^{j}+M \sum_{j=2}^{n} a^{j}\left(w_{1}^{n-j}+w_{2}^{n-j}\right) \\
& \lambda w_{2}^{n}=(n-1+r) w_{2}^{n-1}+\sum_{j=1}^{n-1}\left(\begin{array}{c}
n-1 \\
j-1
\end{array}\right) w_{2}^{j}+M \sum_{j=2}^{n} a^{j}\left(w_{1}^{n-j}+w_{2}^{n-j}\right)
\end{aligned}
$$

$n \geqq 3$

with $w_{0}=\left(\begin{array}{l}1 \\ 0\end{array}\right), w_{1}^{1}=M a^{2}, w_{2}^{1}=0, w_{2}^{2}=\left(M a^{2} / \lambda\right)$, are such that $\left|v_{i}^{n}\right| \leqq$ $w_{i}^{n}, i=1,2, n=0,1, \cdots$, i.e. (4.2) is a dominant system for (4.1). 
Multiplying (4.2) by $a$ and subtracting the resulting equations from (4.2) with $n$ increased to $n+1$ and dropping terms with negative coefficients gives rise to a new dominant system:

$$
\begin{aligned}
n z_{1}^{n}= & a(n-1) z_{1}^{n-1}+\left(\begin{array}{c}
n \\
2
\end{array}\right) z_{1}^{n-1}+\sum_{j=1}^{n-2}\left(\begin{array}{c}
n \\
j-1
\end{array}\right) z_{1}^{j}+M a^{2}\left(z_{1}^{n-1}+z_{2}^{n-1}\right) \\
\lambda z_{2}^{n+1}= & (\lambda a+n+r) z_{2}^{n}+(n-1+r) z_{2}^{n-1}+\left(\begin{array}{l}
n \\
2
\end{array}\right) z_{2}^{n-1}+\sum_{j=1}^{n-2}\left(\begin{array}{c}
n \\
j-1
\end{array}\right) z_{1}^{j} \\
& +M a^{2}\left(z_{1}^{n-1}+z_{2}^{n-1}\right)
\end{aligned}
$$

with the same initial conditions as (4.2). We have

$$
w_{i}^{n} \leqq z_{i}^{n}, \quad i=1,2, \quad n=0,1, \cdots .
$$

Consider $z_{i}^{n} /(n-1)$ ! as dependent variables in (4.3), subtract the corresponding equations from themselves with $n$ increased to $n+1$, and drop all terms with negative coefficients to obtain a new dominant system:

$$
\begin{aligned}
x_{1}^{n+1}= & \left(\frac{a}{n+1}+\frac{3}{2}\right) x_{1}^{n}+\frac{M a^{2}}{n(n+1)}\left(x_{1}^{n}+x_{2}^{n}\right) \\
\lambda x_{2}^{n+2}= & \left(1+\lambda+\frac{\lambda a+r}{n+1}\right) x_{2}^{n+1}+\left(\frac{1}{2}+\frac{a(n+r)}{n(n+1)}\right) x_{2}^{n} \\
& +\frac{M a^{2}}{n(n+1)}\left(x_{1}^{n}+x_{2}^{n}\right)
\end{aligned}
$$

with the same initial conditions as (4.3). We have

$$
z_{i}^{n} \leqq(n-1) ! x_{i}^{n}, \quad i=1,2, \quad n=0,1,2, \cdots .
$$

Since the coefficients in (4.4) are bounded as $n \rightarrow \infty$ we have the estimate

$$
x_{i}^{n} \leqq p e^{n q}
$$

for suitable constants $p$ and $q$. Hence

$$
\left|v_{i}^{n}\right| \leqq w_{i}^{n} \leqq z_{i}^{n} \leqq(n-1) ! x_{i}^{n} \leqq(n-1) ! p e^{n q} .
$$

The same sequence of steps yields similar estimates for the second solution for canonical form (i) and the solutions for canonical forms (ii) and (iv).

We estimate both solutions of canonical form (iii) simultateously due to their interdependence.

The two solutions $u$ and $v$ obtained for canonical form (iii) satisfy the equations 


$$
\begin{aligned}
& v(s+1)=C(s) v(s) \\
& u(s+1)=C(s) u(s)-C(s) \ln \left(1+\frac{1}{s}\right) v(s) .
\end{aligned}
$$

Let $z(s)$ be the vector with four components,

$$
z=\left(\begin{array}{l}
u \\
v
\end{array}\right) \text {. }
$$

Then $z$ satisfies the difference equation

$$
z(s+1)=\widehat{C}(s) z(s),
$$

where

$$
\begin{aligned}
\hat{C}(s) & =\left(\begin{array}{ccr}
C(s) & -C(s) \ln \left(1+\frac{1}{s}\right) \\
0 & C(s)
\end{array}\right) \\
& =I+\frac{1}{s}\left(\begin{array}{rrrr}
0 & 0 & -1 & 0 \\
1 & 0 & 0 & -1 \\
0 & 0 & 0 & 0 \\
0 & 0 & 1 & 0
\end{array}\right)+\frac{1}{s^{2}} \hat{C}_{2}+\cdots .
\end{aligned}
$$

The same sequence of steps (with $a>1$ ) will yield the estimates

$$
\begin{aligned}
& \left|v_{i}^{n}\right| \leqq(n-1) ! p e^{n q} \\
& \left|u_{i}^{n}\right| \leqq(n-1) ! p e^{n q} .
\end{aligned}
$$

To estimate the growth of the coefficients for canonical forms (vii) and (viii) we employ a similar device. We have

$$
\begin{aligned}
v(s)=\sum_{k=0}^{\infty} v_{k} s^{-k / 2} & =\sum_{j=0}^{\infty} v_{2 j} s^{-j}+\frac{1}{s^{1 / 2}} \sum_{j=0}^{\infty} v_{2 j+1} s^{-j} \\
& =u(s)+\frac{1}{s^{1 / 2}} w(s)
\end{aligned}
$$

and

$$
C(s)=\sum_{j=0}^{\infty} C_{2 j} s^{-j}+\frac{1}{s^{1 / 2}} \sum_{j=0}^{\infty} C_{2 j+1} s^{-j}=D(s)+\frac{1}{s^{1 / 2}} F(s) .
$$

Writing

$$
z(s)=\left(\begin{array}{l}
u(s) \\
v(s)
\end{array}\right), \hat{C}(s)=\left(\begin{array}{ll}
D(s) & s^{-1} F(s) \\
\left(1+\frac{1}{s}\right)^{1 / 2} F(s) & \left(1+\frac{1}{s}\right)^{1 / 2} D(s)
\end{array}\right)
$$

we have

$$
z(s+1)=\hat{C}(s) z(s),
$$

where, for canonical form (vii), 


$$
\hat{C}(s)=\left(\begin{array}{cccc}
1 & 0 & 0 & 0 \\
0 & 1 & 0 & 0 \\
\bar{r} & 0 & 1 & 0 \\
0 & 0 & 0 & 1
\end{array}\right)+\frac{1}{s}\left(\begin{array}{cccc}
p & 0 & \bar{r} & 0 \\
0 & 0 & 0 & 0 \\
q+\frac{\bar{r}}{2} & 0 & p+\frac{1}{2} & 0 \\
0 & 0 & 0 & \frac{1}{2}
\end{array}\right)+\frac{1}{s^{2}} \hat{C}_{2}+\cdots,
$$

and for canonical form (viii)

$$
\hat{C}(s)=\left(\begin{array}{rrrr}
-1 & 0 & 0 & 0 \\
0 & 1 & 0 & 0 \\
\bar{r} & 0 & -1 & 0 \\
0 & 0 & 0 & 1
\end{array}\right)+\frac{1}{s}\left(\begin{array}{cccc}
p & 0 & \bar{r} & 0 \\
0 & 0 & 0 & 0 \\
q+\frac{\bar{r}}{2} & 0 & p-\frac{1}{2} & 0 \\
0 & 0 & 0 & \frac{1}{2}
\end{array}\right)+\frac{1}{s^{2}} \hat{C}_{2}+\cdots .
$$

The equations for the components of $z(s)$ will be equivalent to the corresponding equations (3.19)-(3.21) and (3.22)-(3.24) and are given by:

$$
\begin{aligned}
2 z_{1}^{n}= & (p+n-1) z_{1}^{n-1}+\bar{r} z_{3}^{n-1}-\sum_{j=1}^{n-2}(-1)^{n-j}\left(\begin{array}{c}
n-1 \\
j-1
\end{array}\right) z_{1}^{j} \\
& +\sum_{j=0}^{n-2} \sum_{i=1}^{4} C_{1 i}^{n-j} z_{i}^{j}
\end{aligned}
$$

$$
\begin{aligned}
(n-1) z_{2}^{n-1}= & \sum_{j=1}^{n-2}(-1)^{n-j}\left(\begin{array}{c}
n-1 \\
j-1
\end{array}\right) z_{2}^{j}-\sum_{j=0}^{n-2} \sum_{i=1}^{4} C_{2 i}^{n-j} z_{i}^{j} \\
2 z_{3}^{n}= & \frac{\bar{r}}{2} z_{1}^{n}+\left(q+\frac{\bar{r}}{2}\right) z_{1}^{n-1}-\sum_{j=1}^{n-2}(-1)^{n-j}\left(\begin{array}{c}
n-1 \\
j-1
\end{array}\right) z_{3}^{j} \\
& +\left(p-\frac{3}{2}+n\right) z_{3}^{n-1}+\sum_{j=0}^{n-2} \sum_{i=1}^{4} C_{3 i}^{n-j} z_{i}^{j} \\
\left(n-\frac{1}{2}\right) z_{4}^{n-1}= & \sum_{j=1}^{n-2}(-1)^{n-j}\left(\begin{array}{c}
n-1 \\
j-1
\end{array}\right) z_{4}^{j}-\sum_{j=0}^{n-2} \sum_{i=1}^{4} C_{4 i}^{n-j} z_{i}^{j}
\end{aligned}
$$

for canonical form (vii); and

$$
\begin{aligned}
\bar{r} z_{1}^{n}= & -\left(q+\frac{\bar{r}}{2}\right) z_{1}^{n-1}-\left(p+n-\frac{1}{2}\right) z_{3}^{n-1} \\
& +\sum_{j=1}^{n-2}(-1)^{n-j}\left(\begin{array}{c}
n-1 \\
j-1
\end{array}\right) z_{3}^{j}+\sum_{j=1}^{n-2} \sum_{i=1}^{4} C_{3 i}^{n-j} z_{i}^{j}
\end{aligned}
$$

(4.8) $\quad(n-1) z_{2}^{n-1}=\sum_{j=1}^{n-2}(-1)^{n-j}\left(\begin{array}{c}n-1 \\ j-1\end{array}\right) z_{2}^{j}-\sum_{j=0}^{n-2} \sum_{j=1}^{4} C_{2 i}^{n-j} z_{i}^{j}$

$$
\begin{aligned}
\bar{r} z_{3}^{n} & =-(p+n) z_{1}^{n}-\sum_{j=1}^{n-1}(-1)^{n-j}\left(\begin{array}{c}
n \\
j-1
\end{array}\right) z_{1}^{j}-\sum_{j=0}^{n-1} \sum_{i=1}^{4} C_{1 i}^{n-j+1} z_{i}^{j} \\
\left(n-\frac{1}{2}\right) z_{4}^{n-1} & =\sum_{j=1}^{n-2}(-1)^{n-j}\left(\begin{array}{c}
n-1 \\
j-1
\end{array}\right) z_{4}^{j}-\sum_{j=0}^{n-2} \sum_{i=1}^{4} C_{4 i}^{n-j} z_{i}^{j}
\end{aligned}
$$


for canonical form (viii)

In both sets of equations (4.7) and (4.8) the third equation gives $z_{3}^{n}$ in terms of $z_{1}^{n}$ and lower order terms. We could eliminate $z_{1}^{n}$ by use of the first equation. The resulting equation would involve a term essentially $n z_{1}^{n-1}$ for (4.7) and lower terms while the resulting equation would involve a term essentially $n^{2} z_{3}^{n-1}$ for (4.8). The procedure for determining dominate systems of equations breaks down for (4.8) and not for (4.7) due to this fact, i.e. the substitution $z^{n}=$ $(n-1) ! u^{n}$ will give a term corresponding to $n z_{1}^{n-1}$ as a bounded coefficient times $u_{1}^{n-1}$ whereas the term corresponding to $n^{2} z_{3}^{n-1}$ will have a coefficient $0(n)$ times $u_{3}^{n-1}$.

Hence we have, for (4.7) the estimate

$$
\left|z_{i}^{n}\right| \leqq(n-1) ! p e^{n q} .
$$

For (4.8) we do have the estimate

$$
\left|z_{i}^{n}\right|<((n-1) !)^{2} p e^{n q}
$$

but this estimate is too large for subsequent steps in our treatment of the problem under consideration.

REMARK. We note that we have not shown the impossibility of an estimate $\left|z_{i}^{n}\right| \leqq p(n-1) ! e^{n q}$ for canonical form (viii). We are unable to prove such an estimate or construct an example for which $\left|z_{i}^{n}\right|=$ $((n-1) !)^{2} p e^{n q}$. If such an example can be constructed, it is possible to the show the impossibility of summation by the use of factorial series for this example.

5. Related integral equations. We next consider an integral equation for a vector $w(t)$ whose Laplace transform is simply related to our formal solution $v(s)$. We will use this integral equation to show that the formal solution $v(s)$ has a Laplace transform representation $\hat{v}(s) ; v(s)$ is an asymptotic representation of $\hat{v}(s)$; and that $v(s)$ may be summed, i.e. $\hat{v}(s)$ has a convergent factorial series representation.

Consider canonical form (i). We have from (3.3), $v(s+1)=C(s) v(s)$, where

$$
C(s)=\left(\begin{array}{ll}
1 & 0 \\
0 & \bar{\rho}
\end{array}\right)+\frac{1}{s}\left(\begin{array}{cc}
0 & 0 \\
0 & \bar{r}
\end{array}\right)+\frac{1}{s^{2}} C_{2}+\cdots
$$

and the formal solution $v(s)=v_{0}+(1 / s) v_{1}+\cdots$. Formally we may define

$$
w(t)=L^{-1}\left\{v(s)-v_{0}\right\}=L^{-1}\{u(s)\}
$$


Thus, formally, from $v(s+1)=C(s) v(s)$, we have

$$
\left(e^{-t} I-C_{0}\right) w(t)=\int_{0}^{t} \sum_{n=1}^{\infty} \frac{(t-\tau)^{n-1}}{(n-1) !} C_{n} w(\tau) d \tau+\sum_{n=2}^{\infty} \frac{t^{n-1}}{(n-1) !} C_{n} v_{0}
$$

Since $C(s)=\sum_{n=0}^{\infty} C_{n} s^{-n}$ converges for $|s|>s_{0}>0$, the series in (5.2) converge for all finite values of $t$ and $\tau$.

We have from (5.1) that formally

$$
w(t)=\sum_{n=1}^{\infty} \frac{v_{n} t^{n-1}}{(n-1) !}
$$

is a solution of the integral equation (5.2). However, the growth estimate, $\left|v_{i}^{n}\right| \leqq(n-1) ! p e^{n q}$ implies

$$
\left|w_{i}(t)\right| \leqq \sum_{n=1}^{\infty} \frac{\left|v_{i}^{n}\right|}{(n-1) !}|t|^{n-1} \leqq p e^{q} \sum_{n=0}^{\infty}\left(e^{q}|t|\right)^{n}=\frac{p e^{q}}{1-e^{q}|t|}
$$

if $|t|<e^{-q}$. Hence $w(t)$ as given by (5.3) is a true solution since the uniform and absolute convergence of (5.3) for $|t| \leqq \alpha<e^{-q}$ allows the interchange of integration and summation in (5.2).

We now use the integral equation (5.2) to show that $w(t)$ as continued analytically outside the circle $|t|=e^{-q}$ is of exponential order, i.e.

$$
\left|w_{i}(t)\right| \leqq c e^{g|t|}
$$

for positive constants $c$ and $g$ and $t$ in some sector $S$ with vertex at the origin $t=0$.

The singular points for (5.2) are determined by $e^{-t}=1$ and $e^{-t}=$ $\bar{\rho}=|\bar{\rho}| e^{-i \alpha},-\pi<\alpha<\pi$. Thus the possible singular points are

$$
t=2 n \pi i, t=-\ln |\bar{\rho}|+i(\alpha+2 n \pi), \quad n=0, \pm 1, \cdots,
$$

and $w(t)$ can be analytically continued in any sector $\boldsymbol{S}$ which does not contain within or on its boundaries any possible singular point. except $t=0$.

LeMma 2. Let $w(t)$ be defined by

$$
w(t)=\sum_{n=1}^{\infty} \frac{v_{n} t^{n-1}}{(n-1) !}
$$

and satisfy the integral equation (5.2), where $\left|v_{i}^{n}\right| \leqq(n-1) ! p e^{n q}$. Then $w(t)$, with analytic continuation, satisfies the inequality

$$
w_{i}(t) \leqq c e^{g|t|},
$$

$c, g$ positive constants in any sector $\boldsymbol{S}$ which does not contain the 
points

$$
t=2 n \pi i, n= \pm 1, \pm 2, \cdots, t=-\ln |\bar{\rho}|+i(\alpha+2 n \pi), n=0, \pm 1, \cdots .
$$

within or on its boundaries.

Proof. Let $t_{0}$ be such that $0<t_{0}<e^{-q}$ where $e^{-q}$ is less than or equal to the radius of convergence of the series (5.4). Then there exists a constant $c>0$ such that $\left|w_{i}(t)\right|<c$ if $|t|<t_{0}$ and hence $\left|w_{i}(t)\right|<\mathrm{ce}^{g|t|}$ for $|t|<t_{0}$ and $g \geqq 0$.

Assume the lemma false. Then there exists a constant $t_{1}=$ $t_{1}(g)>t_{0}$ such that $\left|w_{i}(t)\right|<c e^{g|t|}$ for $|t|<t_{0}$, while for some point $t^{\prime}$ in $S\left|t^{\prime}\right|=t_{1},\left|w_{i}\left(t^{\prime}\right)\right|=c e^{g t_{1}}$ for $i=1$ or 2 .

Choose as path of integration in (5.2) the ray from the origin thru the point $t^{\prime}$. Evaluating (5.2) for $t=t^{\prime}$ and taking absolute values of the component equations, we have

$$
\begin{aligned}
\left|e^{-t^{\prime}}-1\right|\left|w_{1}\left(t^{\prime}\right)\right| \leqq & \int_{0}^{t_{1}} \sum_{n=2}^{\infty} \frac{\left(t_{1}-\tau\right)^{n-1}}{(n-1) !}\left(\left|c_{11}^{n}\right|\left|w_{1}(\tau)\right|+\left|c_{12}^{n}\right|\left|w_{2}(\tau)\right|\right) d \tau \\
& +\sum_{n=2}^{\infty} \frac{\left|c_{11}^{n}\right|}{(n-1) !} t_{1}^{n-1} \\
\left|e^{-t^{\prime}}-\bar{\rho}\right|\left|w_{2}\left(t^{\prime}\right)\right| \leqq & |\bar{r}| \int_{0}^{t_{1}}\left|w_{2}(\tau)\right| d \tau \\
& +\int_{0}^{t_{1}} \sum_{n=0}^{\infty} \frac{\left(t_{1}-\tau\right)^{n-1}}{(n-1) !}\left(\left|c_{21}^{n}\right|\left|w_{1}\right|+\left|c_{22}^{n}\right|\left|w_{2}\right|\right) d \tau \\
& +\sum_{n=2}^{\infty} \frac{\left|c_{21}^{n}\right|}{(n-1) !} t_{1}^{n-1} .
\end{aligned}
$$

Using $\left|c_{i j}^{n}\right| \leqq M a^{n}, M>0, a>0,\left|e^{-t}-\bar{\rho}\right|>k>0$ for $t$ in $S$ and $\left|e^{-t}-1\right|>k>0$ for $t$ in $S,|t| \geqq t_{0}$, it follows

$$
\begin{aligned}
1 \leqq \frac{|\bar{r}|}{g k}\left(1-e^{-g t_{1}}\right) & +\frac{2 M a}{k}\left(\frac{1-e^{(a-g) t_{1}}}{g-a}-\frac{1-e^{-g t_{1}}}{g}\right) \\
& \left.+\frac{M a}{k c} e^{(a-g) t_{1}}-e^{-g t_{1}}\right)
\end{aligned}
$$

Choose a sequence of $g$ 's, $g=g_{1}, g_{2}, \cdots$ such that $g_{n} \rightarrow \infty$ as $n \rightarrow \infty$. We have $t_{1}\left(g_{n}\right) \geqq t_{1}\left(g_{1}\right)$ and $t_{1}\left(g_{n}\right) g_{n} \rightarrow \infty$ as $n \rightarrow \infty$. The inequality (5.6) holds for $g=g_{n}$, but the limit of the right member of (5.6) as $n \rightarrow \infty$ is zero, a contradiction, and the lemma is proved.

The treatment of canonical form (ii) is similar and the details are omitted.

For the canonical form (iii) we use the four component vector $z(s)$ as given in (4.6). The corresponding integral equation is 


$$
\left(e^{-t}-1\right) w(t)=\int_{0}^{t} \sum_{n=1}^{\infty} \frac{(t-\tau)^{n-1}}{(n-1) !} \widehat{C}_{n} w(\tau) d \tau+\sum_{n=2}^{\infty} \frac{t^{n-1}}{(n-1) !} \widehat{C}_{n} v_{0}
$$

where $w(t)$ is a vector with four components. The possible singular points are $t=2 n \pi i, n=0, \pm 1, \pm 2, \cdots$, and corresponding to Lemma 2 we have

$$
\left|w_{i}(t)\right| \leqq\left(n-1 ! c e^{o|t|}\right.
$$

in any sector $S$ with vertex at the origin which does not contain the rays $\arg t= \pm(\pi / 2)$ within or as boundaries.

For canonical form (iv), the first solution was obtained by the same method used to obtain the solutions for canonical form (i). Thus our treatment for canonical form (i) applies with the restriction $\cos (\arg t)<0$. In order to treat the second solution for canonical form (iv) a modification is necessary.

The second solution for canonical form (iv) satisfies (3.11)

$$
v(s+1)=C(s) v(s)
$$

where

$$
C(s)=s^{m}\left(C_{0}+\frac{1}{s} C_{1}+\cdots\right) \text {. }
$$

Setting $u(s)=v(s)-v_{0}$ and using the relations $C_{0} v_{0}=0, \sum_{n=1}^{m-1}\left(C_{n} / s^{n}\right) v_{0}=0$, we obtain

$$
s^{-m} u(s+1)=C_{0} u(s)+\sum_{n=1}^{\infty} \frac{C_{n}}{s^{n}} u(s)+\sum_{n=m}^{\infty} \frac{C_{n}}{s^{n}} v_{0}-\frac{v_{0}}{s^{m}} .
$$

Formally defining $w(t)=L^{-1}\{u(s)\}$ we obtain

$$
\begin{aligned}
\int_{0}^{t} \frac{(t-\tau)^{m-1}}{(m-1) !} e^{-\tau} w(\tau) d \tau= & C_{0} w(t)+\int_{0}^{t} \sum_{n=1}^{\infty} \frac{(t-\tau)^{n-1}}{(n-1) !} C_{n} w(\tau) d \tau \\
& +\sum_{n=m}^{\infty} \frac{t^{n-1}}{(n-1) !} C_{n} v_{0}-\frac{t^{m-1}}{(n-1) !} v_{0}
\end{aligned}
$$

Defining $w(t)=\sum_{n=0}^{\infty} v_{n+1}\left(t^{n} / n !\right)$, we have that $w(t)$ is a rigorous analytic solution of (5.7) for $|t|<e^{-q}$.

To show that $w(t)$ with analytic continuation satisfies in appropriate sectors $S$ the growth condition $\left|w_{i}(t)\right| \leqq c e^{g|t|}$ we rewrite the integral equation (5.7) in component form and differentiate the equation for the first component $m$ times to obtain 


$$
\begin{aligned}
\left(e^{-t}-1\right) w_{1}(t)= & \int_{0}^{t} \sum_{n=1}^{\infty} \frac{(t-\tau)^{n-1}}{(n-1) !}\left[c_{11}^{n+m} w_{1}+c_{12}^{n+m} w_{2}\right] d \tau+\sum_{n=0}^{\infty} \frac{t^{n}}{n !} c_{11}^{n+m+1} \\
c_{22}^{0} w_{2}(t)= & \int_{0}^{t} \frac{(t-\tau)^{m-1}}{(m-1) !} e^{-\tau} w_{2}(\tau) d \tau \\
& -\int_{0}^{t} \sum_{n=1}^{\infty} \frac{(t-\tau)^{n-1}}{(n-1) !}\left[c_{21}^{n} w_{1}+c_{22}^{n} w_{2}\right] d \tau \\
& -\sum_{n=m}^{\infty} \frac{t^{n-1}}{(n-1) !} c_{21}^{n}+\frac{t^{m-1}}{(m-1) !}
\end{aligned}
$$

Hence $w(t)$ can be continued analytically into either the right or left $t$-plane.

We proceed as in the proof of Lemma 2 and assume that for a sequence of values $g_{n}, g_{n} \rightarrow \infty$ as $n \rightarrow \infty$, either $w_{1}(t)$ or $w_{2}(t)$ violates the growth condition

$$
\left|w_{i}(t)\right| \leqq c e^{g_{n}|t|} .
$$

For $w_{1}(t)$, we use the first equation in (5.8) and the details are similar to those given in the proof of Lemma 2. Hence, assume $w_{2}(t)$ violates the appropriate growth condition. Thus for $g=g_{n}$, using the second equation in (5.8), and $t=t^{\prime},\left|t^{\prime}\right|=t_{1}=t_{1}(g)$, we have

$$
\begin{aligned}
c\left|c_{22}^{0}\right| e^{g t_{1}} \leqq & c \int_{0}^{t_{1}} \frac{\left(t_{1}-\tau\right)^{m-1}}{(m-1) !} e^{-\tau} d \tau+2 M a c \int_{0}^{t_{1}} \sum_{n=0}^{\infty} \frac{\left(t_{1}-\tau\right)^{n}}{n !} a^{n} e^{g \tau} d \tau \\
& +M a \sum_{n=m-1}^{\infty} \frac{t_{1}^{n} a^{n}}{n !}+\frac{t_{1}^{m-1}}{(m-1) !}
\end{aligned}
$$

where $\left|c_{i j}^{n}\right| \leqq M a^{n}, M>0, a>0$.

Integrating the first term of the right member of (5.9) yields

$$
1 \leqq \frac{1}{\left|c_{22}^{0}\right|}\left\{\frac{e^{-t_{1}}}{(g-1)^{m}}+2 M a \frac{1-e^{(a-g) t_{1}}}{g-a}+\frac{M a}{c} e^{(a-g) t_{1}}+\frac{t_{1}^{m-1} e^{-g t_{1}}}{c(m-1) !}\right\} .
$$

The inequality (5.10) holds for $g=g_{n}$, and since $g_{n} \rightarrow \infty$ and $g_{n} t_{1}\left(g_{n}\right) \rightarrow \infty$ as $n \rightarrow \infty$ the right member has limit zero as $n \rightarrow \infty$. Hence we have the desired growth condition

$$
\left|w_{i}(t)\right| \leqq c e^{g|t|}
$$

in any sector $S$ which does not contain the rays $\arg t= \pm(\pi / 2)$ within or on its boundaries.

For canonical form (vii) we utilize the four component vector $z(s)$ used in $\S 4$ to establish the growth estimate $\left|z_{i}^{n}\right| \leqq(n-1) ! p e^{n q}$. The details, being essentially the same as for canonical form (i), are omitted. 
6. Laplace integrals and asymptotic series. For canonical forms (i)-(iv) and (vii) we have shown the corresponding vector $w(t)$ to be of exponential order along rays from the origin in sectors $S$ which contain no singular points. Hence the formal Laplace operator used in $\S 5$ can be put on a rigorous basis.

Select some ray in the sector $S$, say $\arg t=\varphi$. If the complex variable $s=|s| e^{i \theta}$ is restricted to the half plane

$$
H(\varphi): \operatorname{Re}\left\{s e^{i \varphi}\right\}=|s| \cos (\varphi+\theta)>g+\varepsilon,
$$

$\varepsilon>0$ and arbitrary, and the path of integration is along the ray $\arg t=\varphi$, the integral

$$
\widehat{u}(s)=\int_{0}^{\infty} e^{-s t} w(t) d t
$$

converges absolutely in $H(\varphi)$ and defines an analytic vector function. In any sector $S$ (6.1) defines the same analytic vector function, however (6.1) may define different branches of the analytic vector function $\widehat{u}(s)$ in different sectors.

Thus, $\hat{v}(s)=\widehat{u}(s)+v_{0}$ satisfies the difference equation $v(s+1)=$ $C(s) v(s)$, and is a rigorous analytic solution in the union of half-plane $H(\varphi)$ corresponding to rays in the sector $\boldsymbol{S}$.

We may now apply the following theorem of Doetsch [3, p. 231] to show that our formal solutions are asymptotic representations of the solutions $\hat{v}$ in appropriate regions.

THEOREM (Doetsch). Let $\int_{0}^{\infty} e^{-s t} F(t) d t=f(s)$ have a half-plane of conditional convergence and let $F(t)$ have at $t=0$ an asymptotic series expansion

$$
F(t) \sim \sum_{n=0}^{\infty} \frac{c_{n+1} t^{n}}{n !}
$$

Then $f(s)$ possesses in the sector $S:|\arg s| \leqq \beta<(\pi / 2)$ for $s \rightarrow \infty$ the asymptotic series expansion

$$
f(s) \sim \sum_{n=0}^{\infty} \frac{c_{n+1}}{s^{n+1}}
$$

7. Representation of solutions by factorial series. To show that $\widehat{u}(s)$ as given by (6.1), and therefore also $\hat{v}(s)$, is representable by a factorial series we use the following theorem of Nörlund [6, p. 188].

THEOREM (Nörlund). Every integral of the form

$$
u(s)=\int_{0}^{1} z^{s-1} \varphi(z) d z
$$


where the integration path is the real axis, $\varphi(z)$ is an analytic function in the interior of the circle $|z-1|=1$ and of finite order $\omega^{6}$ on this circle, is representable by a factorial series convergent in the half-plane $\operatorname{Re}\{s\}>\omega-1$.

Assume momentarily that the path of integration for $\int_{0}^{\infty} e^{-s t} w(t) d t$ is the positive real axis. Then

$$
\widehat{u}_{i}(s)=\int_{0}^{\infty} e^{-s t} w_{i}(t) d t=\int_{0}^{1} z^{s-1} \varphi_{i}(z) d z
$$

under the transformation $t=\ln (1 / z), w_{i}(t)=\varphi_{i}(z)$. The circle $|z-1|=1$ is mapped onto the $t$-plane as the curve $\sec v=2 e^{v}$ where $t=u+i v$,
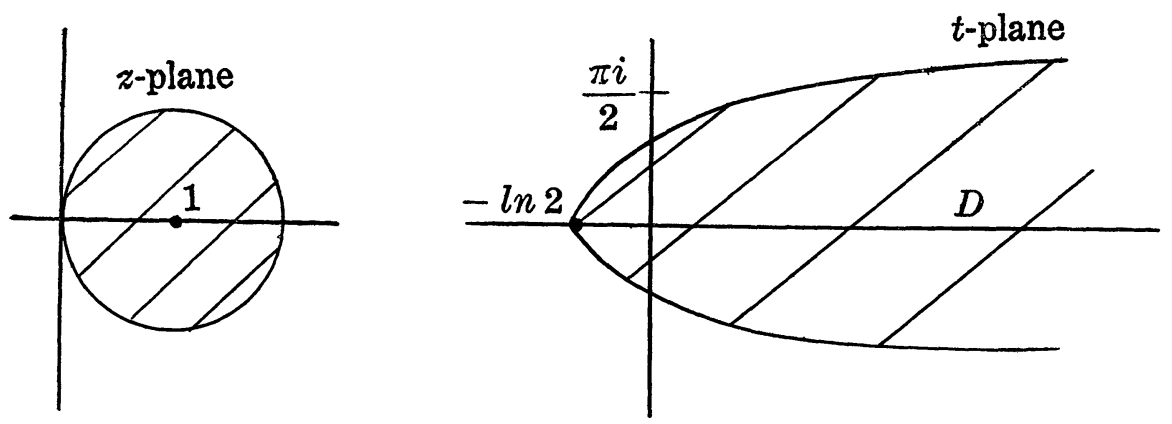

which is asymptotic to the lines $v= \pm(\pi / 2)$. The interior, $|z-1|<1$ is mapped into the region $D$. Assume also that $w_{i}(t)$ is analytic for $t$ in $D$, and hence $\varphi_{i}(z)$ is analytic for $|z-1|<1$. To show that $\varphi_{i}(z)$ is of finite order on $|z-1|=1$ we use the following lemma of Nörlund [6, p. 51].

Lemma (Nörlund). If $(1-|\xi|)^{\omega}|f(\xi)|<C, \omega \geqq 0$, as $\xi$ tends to any point on the circle $|\xi|=1$ from the interior, then the function $f(\xi)$ is of order $\omega+1$ at most on $|\xi|=1$.

Let $z=\xi+1, f_{i}(\xi)=\varphi_{i}(z)=w_{i}(t)=w_{i}(\ln (1 / 1+\xi)) . \quad$ Thus $\left|f_{i}(\xi)\right| \leqq$ $C e^{g|t|} \rightarrow C e^{g\left|l n\left(1+\xi^{\prime}\right)\right|}$ as $\xi \rightarrow \xi^{\prime}$ for $\xi^{\prime} \neq-1,\left|\xi^{\prime}\right|=1$. Hence $\omega \geqq 0$ is sufficient for any point except $\xi^{\prime}=-1$.

Introducing polar coordinates in the $z$-plane we have, $z=r e^{i \theta}$, $r>0,|\theta|<(\pi / 2)$ and for $r<(1 / 2)$

$$
1-|\xi|=r\left(\cos \theta-\frac{r}{2}+\frac{\varepsilon_{\theta}(r)}{r}\right)
$$

${ }^{6}$ Let $f(z)$ be a function defined by a Taylor series $f(z)=a_{0}+a_{1} z+\cdots$ convergent for $|z|<1$. The order $\omega$ of $f(z)$ on $|z|=1$ is $\omega=\limsup _{n \rightarrow \infty} \frac{\ln \left|n a_{n}\right|}{\ln n}$ 
where $\varepsilon_{\theta}(r) / r$ is bounded as $r \rightarrow 0$. Also, given $\varepsilon>0$, for sufficiently small $r,\left|f_{i}(\xi)\right|<C e^{g|b n z|}<C r^{-(1+\varepsilon) g}$. Thus

$$
(1-|\xi|)^{\omega}\left|f_{i}(\xi)\right|<C r^{\omega-(1+\varepsilon) g}\left(\cos \theta-\frac{r}{2}+\frac{\varepsilon_{\theta}(r)}{r}\right)^{\omega}<K
$$

as $r \rightarrow 0$ if $\omega \geqq(1+\varepsilon) g$, and the order of $f_{i}(\xi)$ on the circle $|\xi|=1$ can be of order $g+1$ at most.

In this case we have the factorial expansion

$$
\widehat{u}(s)=\frac{u_{1}}{s}+\frac{u_{2}}{s(s+1)}+\cdots
$$

convergent in the half-plane $\operatorname{Re}\{s\}>g+\varepsilon_{0}{ }^{7}$

In general, let the integration path for $\widehat{u}(s)=\int_{0}^{\infty} e^{-s t} w(t) d t$ be the $\operatorname{ray} \arg t=\varphi$. The analytic solution

$$
\widehat{v}(s)=\widehat{u}(s)+v_{0}
$$

can be represented in the half-plane

$$
H(\varphi): \operatorname{Re}\left\{s e^{i \varphi}\right\}>g+\varepsilon, \varepsilon>0
$$

by the convergent factorial series

$$
\hat{v}(s)=v_{0}+\sum_{k=0}^{\infty} \frac{v_{k+1}(\varphi, \gamma)}{s\left(s+\gamma e^{-i \varphi}\right) \cdots\left(s+k \gamma e^{-i \varphi}\right)}
$$

where the positive constant $\gamma$ is sufficiently large. Any $\gamma>1$ is suitable provided it is large enough so that when the $\xi$-plane is mapped into the $t$-plane by the transformation

$$
t=e^{-i \varphi} \ln \xi / \gamma
$$

the map of the disk $|1-\xi|<1$ is completely contained with a region which is the union of the sector $S$ under consideration and the disk $|t|<e^{-q}$.

We summarize our results in

THEOREM 3. Let the difference equation

$$
y(s+1)=B(s) y(s)
$$

be such that $B(s)$ has one of the canonical forms (i)-(iv) or (vii). There exists a fundamental set of formal solutions of the form

$$
y(s)=s^{n s} \rho^{s} s^{r} v(s), \quad v(s)=\sum_{j=0}^{\infty} v_{j} s^{-j} \quad(\text { (i)-(iv)) }
$$

${ }^{7}$ In some cases the region of convergence may be larger than indicated here. 
or

$$
\begin{aligned}
& y(s)=s^{h s} \rho^{s} \exp \left(\mu s^{1 / 2}\right) s^{r} \exp \left(\nu s^{-1 / 2}\right) v(s) \\
& v(s)=v_{1}(s)+\frac{1}{s^{1 / 2}} v_{2}(s), \quad v_{i}(s)=\sum_{j=0}^{\infty} v_{i j} s^{-j} \quad \text { (vii) }
\end{aligned}
$$

which may be computed by substitution and identification of terms.

These formal solutions are asymptotic representations of true solutions in appropriate regions of the s-plane. Further $v(s), v_{1}(s)$, and $v_{2}(s)$ may be summed and replaced by convergent factorial series in appropriate half-planes of the s-plane.

8. Remarks. (1) Results similar to those of this paper have been obtained by G. D. Birkhoff [1] and W. J. Trjitzinsky [7]. ${ }^{8}$ Both authors consider single linear difference equations of $n$th order

$$
\sum_{k=0}^{n} d_{n-k}(s) y(s+k)=0
$$

where the coefficients are given as convergent power series of the form

$$
d_{n-k}(s)=\sum_{j=-m} d_{n-k_{i j}} s^{-j / p}
$$

where $p$ is an integer. Birkhoff established the existence and form of a full set of formal series solutions; however, his proof was essentially existential and no straight-forward direct method was given to compute successive coefficients in the formal solutions. The summation of these formal series was considered by Trjitzinsky and shown to be feasible in certain cases. Our results are the corresponding results with extensions for linear systems of difference equations with $n=2, p=1$.

Formal solutions of linear systems of difference equations for arbitrary $n$ and $p$ have been considered by H. L. Turrittin [8].

(2) The linear system of difference equations

$$
x(s+1)=A(s) x(s)
$$

where the $n$ by $n$ matrix $A$ has a convergent series representation

$$
A(s)=I+\sum_{k=1}^{\infty} A_{k} s^{-k} \quad|s|>s_{0}
$$

is a special case of a class of linear systems of difference equations solvable by factorial series treated by W. A. Harris, Jr. [4].

(3) General results similar to Lemma 1 are contained in a paper by W. A. Harris, Jr. [5].

\footnotetext{
${ }^{8}$ For other pertinent references see the bibliographies of these two papers.
} 


\section{BIBLIOGRAPHY}

1. G. D. Birkhoff, Formal theory of irregular linear difference equations, Acta Math., 54 (1930), 205-246.

2. W. J. A. Culmer, Convergent solutions of ordinary linear homogeneuus difference equations in the neighborhood of an irregular singular point, $\mathrm{Ph}$. $\mathrm{D}$. thesis, University of Minnesota, 1959.

3. G. Doetsch, Theorie und Anwendung der Laplace-Transformation, Dover 1943.

4. W. A. Harris, Jr., Linear systems of difference equations, Contributions to Differential Equations (to appear).

5. — Equivalent classes of difference equations, Contributions to Differential Equations, 1 (1963), 489-518.

6. N. E. Nörlund, Lesons sur les séries d'interpolation, Gauthiers-Villars, Paris, 1926. 7. W. J. Trjitzinsky, Laplace integrals and factorial series in the theory of linear differential and linear difference equations, Trans. Amer. Math. Soci., 37 (1935), 80-146. 8. H. L. Turrittin, The formal theory of systems of irregular homogeneous linear difference and differential equations, Boletin de la Sociedad Matematica Mexicana, (1960), 255-264.

UNIVERSITY OF MINNESOTA 


\section{PACIFIC JOURNAL OF MATHEMATICS}

\section{EDITORS}

RalPh S. Phillips

Stanford University

Stanford, California

M. G. Arsove

University of Washington

Seattle 5 , Washington
J. Dugundu

University of Southern California

Los Angeles 7, California

Lowell J. Paige

University of California

Los Angeles 24, California

\section{ASSOCIATE EDITORS}
E. F. BECKENBACH
D. DERRY
H. L. ROYDEN
E. G. STRAUS
T. M. CHERRY
M. OHTSUKA
E. SPANIER
F. WOLF

\section{SUPPORTING INSTITUTIONS}

\author{
UNIVERSITY OF BRITISH COLUMBIA \\ CALIFORNIA INSTITUTE OF TECHNOLOGY \\ UNIVERSITY OF CALIFORNIA \\ MONTANA STATE UNIVERSITY \\ UNIVERSITY OF NEVADA \\ NEW MEXICO STATE UNIVERSITY \\ OREGON STATE UNIVERSITY \\ UNIVERSITY OF OREGON \\ OSAKA UNIVERSITY \\ UNIVERSITY OF SOUTHERN CALIFORNIA
}

\author{
STANFORD UNIVERSITY \\ UNIVERSITY OF TOKYO \\ UNIVERSITY OF UTAH \\ WASHINGTON STATE UNIVERSITY \\ UNIVERSITY OF WASHINGTON \\ AMERICAN MATHEMATICAL SOCIETY \\ CALIFORNIA RESEARCH CORPORATION \\ SPACE TECHNOLOGY LABORATORIES \\ NAVAL ORDNANCE TEST STATION
}

Mathematical papers intended for publication in the Pacific Journal of Mathematrcs should be typewritten (double spaced), and the author should keep a complete copy. Manuscripts may be sent to any one of the four editors. All other communications to the editors should be addressed to the managing editor, L. J. Paige at the University of California, Los Angeles 24, California.

50 reprints per author of each article are furnished free of charge; additional copses may be obtained at cost in multiples of 50 .

The Pacific Journal of Mathematics is published quarterly, in March, June, September, and December. Effective with Volume 13 the price per volume (4 numbers) is $\$ 18.00$; single issues, $\$ 5.00$. Special price for current issues to individual faculty members of supporting institutions and to individual members of the American Mathematical Society: $\$ 8.00$ per volume; single issues $\$ 2.50$. Back numbers are available.

Subscriptions, orders for back numbers, and changes of address should be sent to Pacific Journal of Mathematics, 103 Highland Boulevard, Berkeley 8, California.

Printed at Kokusai Bunken Insatsusha (International Academic Printing Co., Ltd.), No. 6 , 2-chome, Fujimi-cho, Chiyoda-ku, Tokyo, Japan.

\section{PUBLISHED BY PACIFIC JOURNAL OF MATHEMATICS, A NON-PROFIT CORPORATION}

The Supporting Institutions listed above contribute to the cost of publication of this Journal, but they are not owners or publishers and have no responsibility for its content or policies. 


\section{Pacific Journal of Mathematics}

\section{Vol. 13, No. $4 \quad$ June, 1963}

Dallas O. Banks, Bounds for eigenvalues and generalized convexity ........... 1031

Jerrold William Bebernes, A subfunction approach to a boundary value problem for

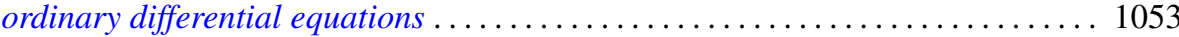

Woodrow Wilson Bledsoe and A. P. Morse, A topological measure construction . . . 1067

George Clements, Entropies of several sets of real valued functions . . . . . . . . . 1085

Sandra Barkdull Cleveland, Homomorphisms of non-commutative *-algebras . . . . . 1097

William John Andrew Culmer and William Ashton Harris, Convergent solutions of

ordinary linear homogeneous difference equations . . . . . . . . . . . . . . . 1111

Ralph DeMarr, Common fixed points for commuting contraction mappings . . . . . . 1139

James Robert Dorroh, Integral equations in normed abelian groups . . . . . . . . 1143

Adriano Mario Garsia, Entropy and singularity of infinite convolutions . . . . . . . 1159

J. J. Gergen, Francis G. Dressel and Wilbur Hallan Purcell, Jr., Convergence of extended Bernstein polynomials in the complex plane ................. 1171

Irving Leonard Glicksberg, A remark on analyticity of function algebras . . . . . . 1181

Charles John August Halberg, Jr., Semigroups of matrices defining linked operators

with different spectra ................................. 1187

Philip Hartman and Nelson Onuchic, On the asymptotic integration of ordinary

differential equations . . . . . . . . . . . . . . . . . . . . . . . . . . . . 1193

Isidore Heller, On a class of equivalent systems of linear inequalities . . . . . . . . . 1209

Joseph Hersch, The method of interior parallels applied to polygonal or multiply

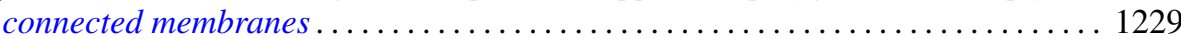

Hans F. Weinberger, An effectless cutting of a vibrating membrane . . . . . . . . . . 1239

Melvin F. Janowitz, Quantifiers and orthomodular lattices ....

Samuel Karlin and Albert Boris J. Novikoff, Generalized convex inequalities . .

Tilla Weinstein, Another conformal structure on immersed surfaces of negative

curvature.

Gregers Louis Krabbe, Spectral permanence of scalar operators

Shige Toshi Kuroda, Finite-dimensional perturbation and a representaion of

scattering operator.

Marvin David Marcus and Afton Herbert Cayford, Equality in certain

inequalities

Joseph Martin, A note on uncountably many disks .

Eugene Kay McLachlan, Extremal elements of the convex cone of semi-norms . . . . 1335

John W. Moon, An extension of Landau's theorem on tournaments . .

Louis Joel Mordell, On the integer solutions of $y(y+1)=x(x$

Kenneth Roy Mount, Some remarks on Fitting's invariants .....

Miroslav Novotný, Über Abbildungen von Mengen ............

Robert Dean Ryan, Conjugate functions in Orlicz spaces.

John Vincent Ryff, On the representation of doubly stochastic operators . . . . . . . . 1379

Donald Ray Sherbert, Banach algebras of Lipschitz functions .

James McLean Sloss, Reflection of biharmonic functions across analytic boundary

conditions with examples.

L. Bruce Treybig, Concerning homogeneity in totally ordered, connected topological space....

John Wermer, The space of real parts of a function algebra...

James Juei-Chin Yeh, Orthogonal developments of functionals and related theorems

in the Wiener space of functions of two variables......... 